\title{
User Behavior during the Book Selection Process ${ }^{1}$
}

\author{
Nina Wacholder \\ School of Communication, Information and Library Studies, Rutgers University. nina@scils.rutgers.edu \\ Lu Liu \\ School of Communication, Information and Library Studies, Rutgers University. Iuliu@scils.rutgers.edu \\ Ying-Hsang Liu \\ School of Communication, Information and Library Studies, Rutgers University. yhliu@scils.rutgers.edu \\ We study user behavior during the stage of the book selection process in which people study \\ the content of a book to decide whether it will be useful for their intended purpose. 24 \\ undergraduates participated in a balanced study in which they were given a topic-book pair and \\ asked to decide whether the book was useful for the topic; we report on the accuracy of the \\ participants' decisions, the extent to which they use the table-of-contents and the index, and the \\ impact of the medium on the book selection process. We discuss barriers to accurate book \\ selection and consider what can be learned, at the applied and theoretical levels, from further \\ study of this activity.
}

\section{Introduction}

We report on an experiment that studies user behavior in the book selection process. This process begins when an individual has an information need that may be satisfied by a book (whether print or electronic) and ends with the user's decision as to whether the book contains enough information on the topic at hand that it merits more-in-depth reading. Our focus is on the stage of the process where the user engages in a combination of information-seeking, navigation and reading to make the decision about the book's usefulness.

The objectives of this research are to find out how well people do at this 'real-world' information access task and to understand what actions people take to make the decision as to whether or not a non-fiction book is useful for a particular topic. How accurate are the users' decisions? To what extent do users make use of the table-of-contents, the index, and, in digitized books, the search function? What is the relative usefulness of these tools? What impact does the medium (electronic vs. print) have on the book selection process?

We conducted a balanced experiment in which we presented participants with a book and a topic and asked them to decide whether the book would be useful for writing a paper on this topic. We measure the outcome of book selection in terms of the accuracy of participants' decisions about the usefulness of a book for a particular topic, the time the participant took to make the usefulness decision and the pattern of page navigation. We measure the impact of usefulness of the topic, medium of presentation of the text, and participants' perception of the difficulty of the book and the topic.

\section{The book selection process}

Traditionally, book selection by library clientele, students and researchers has taken place in locations where physical books are available for inspection. We note that book selection by users that we discuss in this paper is distinct from the book selection that librarians engage in when they acquire

\footnotetext{
${ }^{1}$ This work was funded by NSF grant \# 0414557, Michael Lesk and Nina Wacholder, Pls.
} 
books for their institutions. But as a result of the extensive digitization of printed books by the Million Book Project ${ }^{2}$, Google Book Search ${ }^{3}$ and the Open Content Alliance ${ }^{4}$ and of the book selection interfaces offered by online booksellers such as Amazon ${ }^{5}$, book selection has been added to the list of information access activities that can be accomplished with physical or virtual text.

Book selection can be characterized as a complex fusion of three inter-related information access activities, each of which has been studied separately: 1) information-seeking; 2) browsing or navigating and 3 ) reading.

In information-seeking (e.g., Marchionini 1995, Belkin 1993), the user identifies an information need, develops a strategy to find information that satisfies the need, and responds to the outcome of a step in the process either by reiterating the search process or by stopping. To make the decision that a book is useful, the user has to find evidence of some relatively substantial amount of relevant content. To make the decision that a book is not useful, the user has to get enough of an overview of the book to be confident that the book does not have useful content. The user may obtain this information by stringsearching, by referring to the table-of-contents or index, by browsing and/or by reading all or part of the book.

The fundamental measure of the success of the information-seeking process is the outcome, i.e., whether the information seeker has found the requisite information. But outcome is not all that matters - process is also important because of its potential impact on the success of the information-seeking process and also because users prefer to exert as little effort in the process as possible.

In browsing, which is sometimes called navigating and is sometimes considered part of the informationseeking process, the user moves back and forth in a book, either by turning pages or by using hypertext links (e.g., Catledge \& Pitkow, 1995; Bates 1989, Marchionini \& Schneiderman 1988). The user may browse to understand more about the information need or topic at hand, or to get an overview of the organization of the text and of the author's approach.

Reading takes place when the user visually processes some part of the text of the book and engages in mental activity that results in comprehension. The book selection task primarily demands what we call 'spotty reading', a process in which readers do not read the pages of a book consecutively, from beginning to end; instead they read a section of text and then jump to another part of the book. Spotty reading is especially common in reading of non-fiction and in doing research. For example, Summerfield and her colleagues (1999) report that $25 \%$ of their readers moved forward through online books from the beginning while the remaining $75 \%$ skipped around. We assume that in general spotty reading requires more cognitive effort than does reading for simple fact seeking but less effort than does reading for comprehension.

Speed and accuracy are two measures used to assess the outcome of the reading process; it is desirable also to use comprehension, but it is very hard to measure and so there have been relatively few experiments (Dillon, 2004). Researchers sometime try to measure comprehension based on accuracy, but accuracy on a task and comprehension are not the same.

We adopt measures used in the study of information-seeking, browsing and reading to the book selection process.

\footnotetext{
${ }^{2}<$ http://www.archive.org/details/millionbooks>

${ }^{3}<$ http://books.google.com/>

${ }^{4}$ http://www.opencontentalliance.org/

${ }^{5}$ http://www.amazon.com/
} 


\section{Study Design}

To observe the process of non-fiction book selection as users typically engage in it, we designed an experiment with tasks, users and systems as realistic as we could reasonably make them.

1. TASK. In addition to being a core activity of library users, the fact that book selection is a fairly standard undergraduate assignment contributes to the fundamental reality of the task. On the other hand, two unavoidable divergences from reality follow from our decision to conduct a controlled experiment. First, participants were not performing the task as a graded effort; this may have lessened their motivation. Second, participants did not have the background in British history that they would have had if they were actually enrolled in a class on that subject. Within these limitations, we selected books and topics that would be accessible and interesting to undergraduates.

We downloaded seven books from the Million Book Project web site (one for training and 6 for testing) that met the following criteria: 1) They were general texts about $19^{\text {th }}$ century British history; 2) They included a table of contents and an index; 3) They were relatively readable; in practice this meant that we eliminated books with characteristics such as a dull writing style that would be off-putting to students; and 4) The scan provided by the Million Book Project was of relatively good quality. All of the books are out-of-copyright and were published between 1898 and 1937 . They ranged in length from 255 to 526 pages; the mean was about 430 pages. A list of the books used in the experiment is in Appendix A.

Since not every book is helpful for every topic, we designed two topics for each book: one was useful and the other was not. Because our subjects were undergraduates, we tried to pick topics that were not too specific or technical. Since searching of electronic text is better for tasks where the questions use the author's vocabulary (Egan et al., 1989) we deliberately used wording different than that of the author. Topics were composed with the book in-hand so that the usefulness judgments were correct. A professor of British history reviewed each topic for reasonableness.

As an example, the useful topic for the book England Since Waterloo was:

For a paper on social and economic class in 19th century Britain, collect information about different social and economic groups and about factors that affected the well-being of one or more such groups.

To decide that a book was useful, the participant had to find at least one chunk of promising text.

An example of a topic for which England Since Waterloo did not contain helpful information was:

For a paper on religious freedom and tolerance in 19th century England, collect examples or evidence of religious freedom and tolerance or the lack thereof.

The rest of the books and topics are in Appendix B.

Making the decision that a book is not useful can be considerably harder - the user has to correctly judge that useful text cannot be found, even in the parts of the book they didn't look at. So it is particularly important for these topics that the reader get an overview of the content of the book.

2. USERS. The 24 participants in the study were undergraduates at a large state university. The students, mostly majors in communication or information technology, are by and large technologically proficient but do not have the domain expertise to be sophisticated assessors of the usefulness of books. The participants constitute a convenience sample recruited by fliers and email and through experimenter visits to undergraduate classes. We required that participants have a Western high school education so that they had at least some exposure to British history. Appendix B summarizes the demographic information. We note that although information about the students' academic performance or intelligence would have been helpful, we did not collect this information for the present 
study; self-reporting is not trustworthy and we were not sure whether the effort to collect this data accurately would be worthwhile for this experiment.

3. SYSTEM. Each book was prepared for presentation to the participants in two formats, PDF and print. PDF was chosen because of it is readily available and familiar to most students and because it preserves the book layout. The paper copy was printed out from the PDF version and spiral-bound so that it lay flat during use. The printed version was prepared on a professional quality printer; the electronic version was displayed on a mid-grade Dell laptop. We judged the quality of both displays to be adequate. Besides the physical differences associated with the two media, the main difference between the two information systems was the availability of the search function in PDF; only single word searches were supported.

\section{Procedure}

During the one and a-half hour sessions, participants engaged in the following activities:

- Filling out a demographic survey (5 mins.).

- Training, including a review of PDF and a practice topic using a book not part of the actual study; experimenters used a script so that all participants got the same instructions (10 mins.).

- Determining the usefulness of 6 topics, each for a different book; a maximum of 10 minutes was allotted to each book-topic pair (60 mins.).

- Completing a post-topic questionnaire that asked about the difficulty of the book, the difficulty of the topic, and the participant's confidence in the correctness of the answer (5 mins.).

- Reporting to the interviewer about which medium they preferred (10 mins.).

Each subject saw each book only once; a Latin square design was used to control assignment of books, medium and topic. Half of the subjects saw the PDF first; half saw the print versions first.

To try to understand the book selection process, we collected data about the pages participants read and the order in which they read the pages. For PDF users, page numbers and order of pages viewed were logged by the system; a page number was recorded if it remained open on the screen for 3 seconds or more. Users of the printed text received a stack of sticky notes had been consecutively numbered in advance of the session and were instructed to attach one to each page they read. If they read a page more than once, they were to use multiple notes. The experimenter reminded participants to insert sticky notes if they seemed to forget do so; one result of this procedure is that the number of pages read by paper users may be under-reported.

To find out what pages participants used to make their decision about book usefulness for a topic, we gave participants a form on which we asked them to record pages that helped them make their decision about usefulness while they were inspecting the book. Immediately after recording their decision for each book-topic pair, participants answered questions about their confidence in the correctness of their usefulness judgment and about the difficulty of the book and the topic.

The experimenter sat in on the session, kept times and was available to help participants with procedural problems.

\section{Results}

We used both outcome and process measures for this initial study. The dependent variables are 1) Accuracy of the decision about book usefulness, a measure of the effectiveness of the book selection 
process. 2) Time spent, a measure of efficiency indicating how much effort the process demands from the participants' and 3) Lists of pages read and the order in which they were provided, which represent the participants' navigational process.

Looking at the dependent variables in isolation provides an overview of the difficulty of the task and the participants' engagement in the experiment.

1. EFFECTIVENESS. Of the 144 data points (24 users * 6 books), 50\% (72) of the answers were correct, $29 \%$ (42) were incorrect, and participants were unsure in $21 \%(30)$ cases. If the incorrect and unsure cases are combined, $50 \%$ of the answers were correct and $50 \%$ were incorrect. No participants got 6 correct answers and only 6 participants got 4 or 5 answers right; 18 participants got half or less of the answers correct (Table 1).

Table 1. Number of participants obtaining each possible number of correct answers

\begin{tabular}{|c|c|}
\hline Correct answers & Number of participants \\
\hline 6 & 0 \\
\hline 5 & $3^{*}$ \\
\hline 4 & $3^{*}$ \\
\hline 3 & $10^{* *}$ \\
\hline 2 & $7^{* *}$ \\
\hline 1 & $1^{* *}$ \\
\hline 0 & 0 \\
\hline
\end{tabular}

*‘Good' performers; **'Not-so-good’ performers

We refer below to the users who got 4 or more correct answers out of 6 as the 'good' performers and to the users who got less than 4 answers correct as the 'not-so-good' performers.

This data indicates that the book selection task as operationalized in this experiment is indeed relatively difficult for most of these users. We attribute this to the fact that the participants were non-history majors (except for one), the texts' age was reflected in a distinctly old-fashioned writing style and the wording of the topics was designed to make simple string-searching hard.

2. TIME. Participants spent an average of 7.8 minutes (out of 10 minutes allotted) on each subjecttopic pair.

Table 2: Average time spent on book-topic pair by participants

\begin{tabular}{|c|c|c|}
\hline \multicolumn{2}{|c|}{ Minutes } & $\begin{array}{c}\text { Number of } \\
\text { Participants }\end{array}$ \\
\hline At least & Less than & 3 \\
\hline 10 & & 3 \\
\hline 9 & 10 & 4 \\
\hline 8 & 9 & 8 \\
\hline 7 & 8 & 1 \\
\hline 6 & 7 & 5 \\
\hline 5 & 6 & 0 \\
\hline
\end{tabular}


The fact that no participant spent an average of less than 5 minutes per topic-pair is confirming evidence that the task was hard. It also suggests that participants were motivated to find the right answer; if they had been "blowing off" the task, they presumably would have spent less time on each book-topic pair. The experimental observers confirm that the participants were actively engaged in the task.

3. PAGES LOOKED AT. On average the participants looked at about 16 pages; the average book length was about 430 pages. So by this measure participants made their decision about usefulness by looking at under $4 \%$ of the book and looked at about 2 pages per minute (16 pages / 8 minutes). We recognize that without comparison data, these numbers are impossible to interpret; we report this number here because it may be of interest for future study of similar tasks.

\section{Barriers to effective and efficient book selection}

We focused on several possible barriers to the book selection process: 1) usefulness of the book for the topic; 2) medium of presentation; and 3) participant's judgment of book difficulty and of topic difficulty. These are the independent variables.

1. USEFULNESS OF BOOK FOR TOPIC. Whether a book was 'on' or 'off' topic did affect the accuracy of participants' decisions about book usefulness: subjects tended to make significantly more correct answers, at the .05 level, when the book was off-topic $\left(x^{2}=8.95\right.$; $\left.d f=2 ; p=0.01\right)$. We speculate that this is because it is harder to make an accurate judgment that a book is off-topic than that it is on-topic. To make the on-topic judgment, the user has a) to find a place that has useful information and b) to recognize that information as useful. If either of these conditions is not met, the user will decide that a book is not useful. This suggests that in real life, people engaged in the book selection task miss many useful books from among those that they have looked at. This is the book selection equivalent of the recall problem, which has been documented at least since Blair and Maron (1985) showed that the attorneys who used the STAIRS document-retrieval system thought that they had achieved a much higher level of recall than they actually did.

2. MEDIUM OF PRESENTATION. Although medium of presentation did not have a significant relationship to correctness, participants made more accurate decisions about book usefulness when they worked with the paper version of the book than with the electronic version. For example, with paper, 56\% of judgments were correct and $44 \%$ incorrect; with PDF, only $44 \%$ of accuracy judgments were accurate and $35 \%$ were inaccurate.

Interestingly, the not-so-good performers did considerably better with print than with PDF (Table 3); medium of presentation made a difference with a significance level of $0.1\left(x^{2}=4.7, d f=2, p\right.$-value $=$ 0.0938).

Table 3: Accuracy judgments of 18 not-so-good performers

\begin{tabular}{|c|c|c|}
\hline & Print & PDF \\
\hline Accurate (Usefulness rating of 4 or 5) & 28 & 17 \\
\hline Inaccurate (Usefulness rating of 1 or 2) & 15 & 23 \\
\hline Unsure (Usefulness rating of 3) & 11 & 14 \\
\hline
\end{tabular}

But for the good performers, medium was not related to accuracy of results (Table 4).

Table 4: Accuracy judgments of 6 good performers

\begin{tabular}{|c|c|c|}
\hline & Print & PDF \\
\hline Accurate (Usefulness rating of 4 or 5 ) & 12 & 15 \\
\hline Inaccurate (Usefulness rating of 1 or 2 ) & 2 & 2 \\
\hline
\end{tabular}




\section{Unsure (Usefulness rating of 3)}

\begin{tabular}{l|l|}
4 & 1
\end{tabular}

Good performers did slightly better with PDF than with print, but the difference is not significant $\left(x^{2}=\right.$ $2.1, \mathrm{df}=2$, $\mathrm{p}$-value $=0.3442$ ).

Obviously it is possible to make accurate decisions about book usefulness with either medium. We speculate that the book selection task as operationalized in this experiment is easier to perform accurately with print than with PDF, but that the 'good' performers were better able to compensate for the limitations of PDF.

We also observed that although the average number of pages read by users of PDF and print versions of the books did not differ significantly, the parts of the book they looked at do differ, as shown in Table 5 .

Table 5: Number of pages read by 24 subjects

\begin{tabular}{|c|c|c|c|}
\hline Average number of pages read per topic in: & Print & PDF & p-value of t-test \\
\hline Entire book & 16.2 & 15.4 & 0.3794 \\
\hline Prefatory content & 0.8 & 1.2 & 0.1181 \\
\hline Table-of-contents & 7.2 & 3.2 & $1.28 \mathrm{e}-8^{*}$ \\
\hline Main text & 6.4 & 10.3 & $2.46 \mathrm{e}-5^{\star}$ \\
\hline Index & 1.8 & 0.6 & $0.00016^{*}$ \\
\hline
\end{tabular}

*Significant at .05 level

These patterns can also be seen in Figures 1 and 2 .

These results show that the availability of the search function in the PDF version affects the parts of the book the participants looked at. But at least for the not-so-good performers, the impact was not beneficial. We posit several possible reasons: 1) Differential use of table-of-contents and index. 2) It's harder to get an overview of a book by searching than by leafing through pages; 3) Participants read less of the text when they were searching; this may be because when people search they focus on the target string and the immediate environment but not the larger context. 


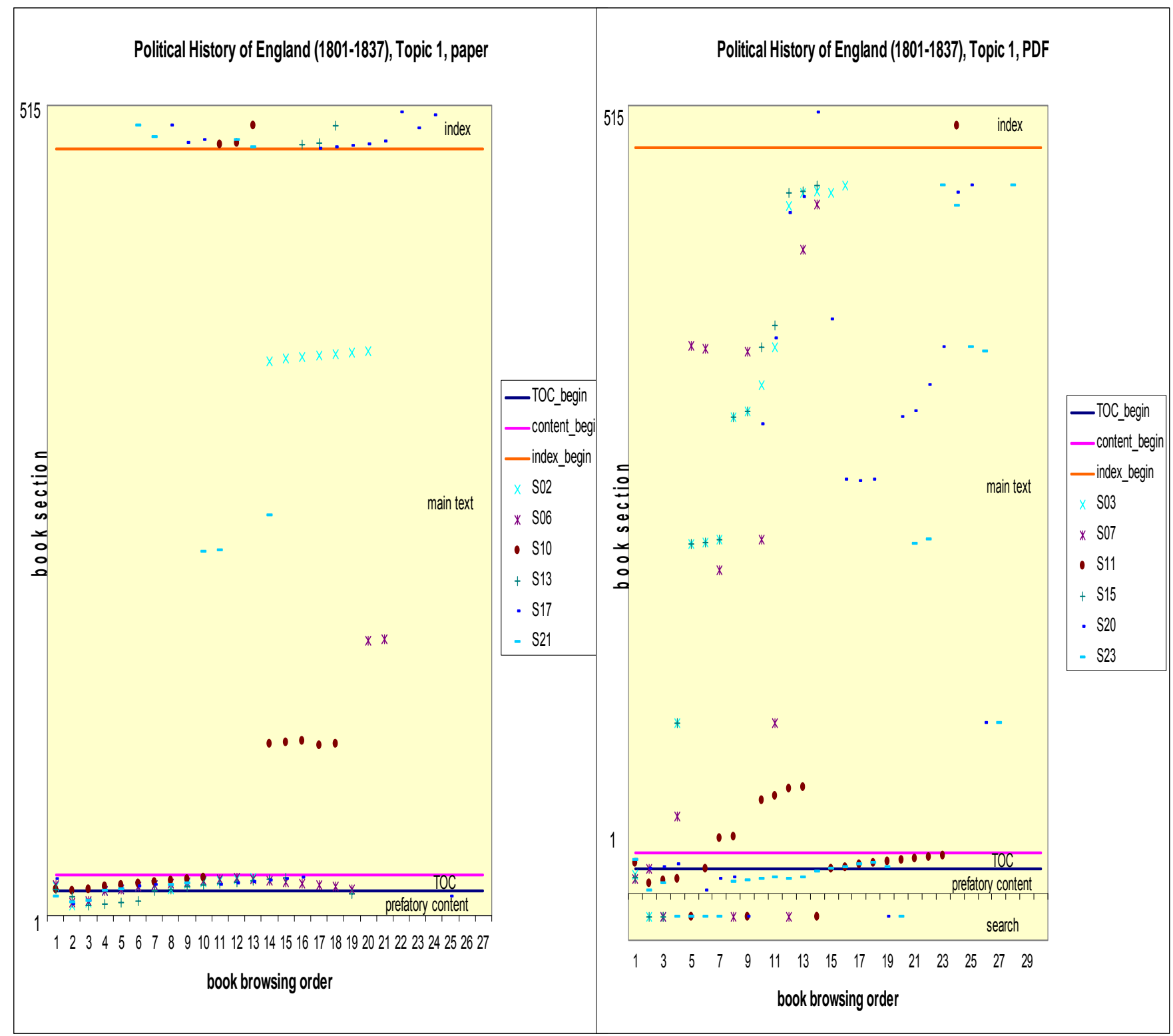

Figure 1. Political History of England, paper

Figure 2. Political History of England, PDF

3. PARTICIPANTS' PERCEPTION OF BOOK AND TOPIC DIFFICULTY. To understand how difficulty measures are related to answer accuracy, average time spent, and average number of pages viewed, we used multivariate analysis. Neither participants' perception of book difficulty nor their perception of topic difficulty was related to answer accuracy. Book difficulty did have a positive relationship with average time spent and average number of pages viewed, though topic difficulty did not. This is shown in Table 6 and Table 7.

Table 6: Book/topic difficulty and average time spent

\begin{tabular}{|c|c|c|c|c|c|}
\hline Source & SS & df & MS & F & P \\
\hline Book difficulty & 48.26 & 1 & 48.26 & 9.88 & 0.002 * \\
\hline
\end{tabular}




\begin{tabular}{|c|c|c|c|c|c|c|}
\hline Topic difficulty & 4.50 & 1 & 4.50 & 0.92 & 0.339 \\
\hline Residuals & 688.71 & 141 & 4.88 & & \\
\hline
\end{tabular}

Table 7: Book/topic difficulty and average number of pages viewed

\begin{tabular}{|c|c|c|c|c|c|}
\hline Source & SS & Df & MS & F & p \\
\hline Book difficulty & 283.8 & 1 & 283.8 & 8.71 & $0.004^{*}$ \\
\hline Topic difficulty & 24.9 & 1 & 24.9 & 0.77 & 0.38 \\
\hline Residuals & 4591.9 & 141 & 32.6 & & \\
\hline
\end{tabular}

$\mathrm{SS}=$ sum of squares, $\mathrm{df}=$ degree of freedom, MS = mean square. *Significant at .05 level

Number of pages looked at and time spent are measures of effort expended, so we speculate that participants judged books to be difficult if they felt that they had exerted a lot of cognitive energy in making the usefulness decision. We were surprised not to find a similar impact for topic difficulty, but if there are observable impacts of participants' perception of topic difficulty, we were unable to identify them.

In term of perceptions of book and topic difficulty, good performers judged the topics and the books to be significantly less difficult than the not-so good performers did at the $5 \%$ significance level $(t=-2.43$; df $=69.65, p=0.017)$; they judged the topics less difficult at the $10 \%$ significance level $(t=-1.99 ; d f=69.65$; $\mathrm{p}=0.051)$.

\section{DISCUSSION AND CONCLUSION}

Our findings about the book selection process include the following:

- Participants engaged energetically in the book selection task, even though they found it difficult. Because this task has a measurable outcome based on a priori judgments of book-topic usefulness, this realistic task is appropriate for comparing the usefulness of electronic book viewers.

- The distinction between good and not-so-good performers is apparent in the less successful use of digitized books by the not-so-good performers. If this result is confirmed by additional studies, it is desirable to design electronic book viewers that help with this process. We note that we studied only books digitized as PDF; more sophisticated electronic book viewing tools are available and some of these may be better for this purpose than PDF. Librarians and information educators should also take these differences in the impact of medium of presentation into account for user instruction.

The explanation for the different performance of good and not-so-good performers associated with different media may be related to different searching ability and/or different kinds of use of tables-ofcontents and indexes. Study of the book selection process that focuses on the different contributions of these different 'text entry' points may help to better understand how they are used for real world tasks and how their design can be improved.

- From the fact that participants' perception of book difficulty was related to time spent and pages viewed during the book selection process, we deduce that judgment of book difficulty is based on effort expended. We do not have an explanation of what caused participants to label certain topics as difficult.

Because these conclusions are based on a single study, we refrain from generalizing to book selection in general. However, we anticipate that these results will be replicated and extended by studies using different kinds of books, different topics and different information systems. 
A regrettable limitation of this study is the absence of adequate data about participants' academic backgrounds and proficiency. The focus of this paper was on barriers in the information access process itself that limit successful book selection; however, more information about individual differences is needed for future studies.

One disappointment of this research was our inability to find navigational patterns that distinguish ontopic and off-topic books and only limited patterns that distinguish book selection of electronic and print books. The decision to study book selection in printed books limited the data we could collect about navigation; for example, we had to rely on sticky notes inserted by participants to keep track of pages participants looked at. We conjecture that use of statistically advanced techniques for identifying patterns in navigating hypertext on detailed logs obtained from digital book selection may be more productive.

The observations reported here are examples of what can be learned from research on the book selection process and provide some lessons for future studies. Perhaps as important as these observations are the avenues of research that this study suggests are promising. These include the following:

- Repeating this experiment with books from different domains, with harder and easier topics. and with harder and easier books.

- Comparing the outcome of the book selection process in books with and without a table-of-contents and with and without indexes.

- Collection and analysis of more detailed logs that capture details of the book selection process.

- Investigation of the effect of individual differences such as areas of academic interests and ability on the book selection process.

- Investigation of the impact of the user's mental model of conventional book structure and its carryover to the digital environment.

- Qualitative study of individuals engaged in the book selection process.

\section{CONCLUSION}

This study furthers our understanding of the barriers that impede accurate book selection and of how to overcome these barriers. At the applied level, this research has implications for the design and evaluation of systems that help users access text content and for user instruction. At the theoretical level, this research contributes to our understanding of a fundamental information access process. More research is needed to provide a broader context for our findings.

\section{REFERENCES}

Bates, M. J. (1989). The design of browsing and berrypicking techniques for the online search interface. Online Review, 13(5), 407-424.

Belkin, N. J. (1993). Interaction with texts: Information retrieval as information-seeking behavior. In Information Retrieval '93: Von der Modellierung zur Anwendung (pp. 55-66). Konstanz:

Universitätsverlag Konstanz.

Blair, D. C., \& Maron, M. E. (1985). An evaluation of retrieval effectiveness for a full-text documentretrieval system. Communications of the ACM, 28(3), 289-299. 
Catledge, L. D., \& Pitkow, J. E. (1995). Characterizing browsing strategies in the World-Wide Web. Computer Networks and ISDN Systems, 27(6), 1065-1073.

Dillon, A. (2004). Designing usable electronic text (2nd ed.). Boca Raton, FL: CRC Press.

Egan, D. E., Remde, J. R., Gomez, L. M., Landauer, T. K., Eberhardt, J., \& Lochbaum, C. C. (1989). Formative design-evaluation of SuperBook. ACM Transactions on Information Systems, 7(1), 30-57.

Marchionini, G. (1995). Information seeking in electronic environments. New York: Cambridge University Press.

Marchionini, G., \& Shneiderman, B. (1988). Finding facts vs. browsing knowledge in hypertext systems. Computer, 21(1), 70-79.

Summerfield, M., Mandel, C., \& Kantor, P. (1999). The Online Books Evaluation Project final report. Retrieved June 22, 2006, from http://www.columbia.edu/cu/libraries/inside/projects/olb/olbdocs/finalreport.pdf

\section{APPENDIX A: Books and topics used in the experiment}

1. Marriott, J. A. R. (1913). England since Waterloo (13th edition, 1944). London: Methuen \& Co. LTD. Topic for which book is useful: For a paper on social and economic class in 19th century Britain, collect information about different social and economic groups and about factors that affected the well-being of one or more such groups.

Topic for which book is not useful: For a paper on religious freedom and tolerance in 19th century England, collect examples or evidence of religious freedom and tolerance or the lack thereof.

2. Hunt, W. (1905). The history of England: From the accession of George III to the close of Pitt's First Administration, 1760 - 1801. London: Longmans, Greens, and Co.Davies,

Topic for which book is useful: What kind of policies did the British government impose on its colonies with regard to their local government and personal freedom? How were these policies similar to or different than British policies regulating the freedom of the colonies to engage in trade with other countries?

Topic for which book is not useful: What was the effect of social class upon the Army and Navy? Did the British social system affect the fighting abilities of its military forces?

3. McCarthy, J. (1898). The story of the nations: Modern England before the Reform Bill. London: T. Fisher Unwin, Paternoster Square.

Topic for which book is useful: In what ways was freedom of the press restricted in England in the first half of the 19th century?

Topic for which book is not useful: What progress did industrialization make in Britain in the first half of the 19th century? What sources of capital were available, and what government programs encouraged the rise of industry?

4. Marriott, J. A. R. (1909). The remaking of modern Europe: From the outbreak of the French Revolution to the Treaty of Berlin, 1789 - 1878 (24th edition, 1946). London: Methuen \& Co. LTD. Topic for which book is useful: Your assignment is to write a paper about how the French revolution affected the spread of democracy in Europe. A useful book will contain either examples or discussion of this issue. Note that a brief mention of the fact that democracy did spread is not enough to make the book useful. 
Topic for which book is not useful: Your assignment is to write a paper about the relationship between the slave trade and the political and military turmoil in France in the first half of the 19th century. A useful book will explore one or more aspects of the slave trade and provide examples of the relationship or discussion of the issues. Note that a brief mention of the existence of slave trade is not enough to make the book useful.

5. G. (1937). The Early Stuarts, 1603 - 1660. London: Oxford.

Topic for which book is useful: Your assignment is to write a paper about freedom of religion (or lack thereof) in 17th century England. A useful book will include evidence or discussion about laws that relate to religious freedom and/or religious tolerance.

Topic for which book is not useful: Your assignment is to write a paper about the evolution of naval technology. What improvements in naval architecture were made in the early 17th century? How were the wars with European countries affected by changes in shipbuilding?

6. Brodrick, G. C., \& Fotheringham, J. K. (1919). The history of England: From Addington's Administration to the close of William IV's reign, 1801 - 1837. London: Longmans, Greens, and Co. Topic for which book is useful: For a paper on British colonialism in the first part of the 19th century, collect examples of the discontent of the people who lived in the British colonies.

Topic for which book is not useful: Industrial revolution begun in Britain led to free competition in European economy. On the other hand, monopolies began to appear in order to control the market. What were the types of monopolies or industrial combinations in the 19th century? What was the attitude in Britain toward those industrial combinations?

7. George, D. M. (1925). London life in the 18th century. London: Kegan Paul, Trench, Trubner and Co. Reprinted in 1976 by Penguin Books. (Training text.)

Topic for which book is useful: What kind of education did poor children receive in England in the 18th century?

Topic for which book is not useful: How common was consumption of alcohol in Europe in the 18th century? Collect information about attitudes towards alcohol consumption and its impact on society.

\section{APPENDIX B: Demographic data on participants}
Population:
24 undergraduates
14 male; 10 female
Computer use the previous day
3.3 hours (mean)
Comfort using table-of-contents \& index
Comfort using PDF
$4.7^{*}$
Comfort reading on-screen
$4.2^{*}$
$3.5^{*}$

* 1 is lowest; 5 is highest 